\title{
Comment ne pas être religieux? Feuerbach et Marx : esquisse de phénoménologie socio- historique du religieux
}

\section{Natalie Depraz}

\section{OpenEdition}

Journals

Édition électronique

URL : https://journals.openedition.org/alter/2012

DOI : $10.4000 /$ alter.2012

ISSN : 2558-7927

Éditeur :

Association ALTER, Archives Husserl (CNRS-UMR 8547)

\section{Édition imprimée}

Date de publication : 1 novembre 2020

Pagination : $97-115$

ISBN : 978-2-9550449-6-4

ISSN : 1249-8947

Référence électronique

Natalie Depraz, "Comment ne pas être religieux ? Feuerbach et Marx : esquisse de phénoménologie socio-historique du religieux », Alter [En ligne], 28 | 2020, mis en ligne le 22 décembre 2020, consulté le 14 octobre 2021. URL : http://journals.openedition.org/alter/2012; DOI : https://doi.org/10.4000/alter. 2012

Ce document a été généré automatiquement le 14 octobre 2021.

Revue Alter 


\title{
Comment ne pas être religieux? Feuerbach et Marx : esquisse de phénoménologie socio-historique du religieux
}

\author{
Natalie Depraz
}

La religion, cette névrose obsessionnelle universelle de l'humanité Freud, L'avenir d'une illusion (1927)

\section{Introduction}

«Sartre est philosophique de l'Occident ${ }^{1}$. C'est le philosophe et théologien Christos Yannaras qui s'exprime. Provocation? Plutôt : vertu de l'athéisme de l'auteur de L'être et le néant qui, bel héritier de Nietzsche et de Heidegger, produit un diagnostic au scalpel de la posture religieuse molle du piétisme du XIX ${ }^{\mathrm{e}}$ siècle.

Dans cette contribution, je montrerai comment le rapport de l'humain à la religion contient une double postulation: une nécessité et un questionnement. La nécessité repose sur la double négation d'un « ne pas pouvoir ne pas croire »; le questionnement opère une démarcation entre la religion opium du peuple et la religion ouverture du soi à autre que soi.

Depuis une enquête généalogique étayée par la méthode de l'épochè et de la variation eidétique, je dégagerai trois propriétés du mode d'être religieux. Puis je mettrai ces propriétés à l'épreuve de la sape la plus radicale du religieux qui ait été opérée au XIX ${ }^{\mathrm{e}}$ siècle, pour tester leur capacité de résilience. La question sera: pourquoi l'humain même athée a-t-il toujours, encore pour destin le religieux? 


\section{I. Éléments de phénoménologie du religieux}

\section{Le religieux et l'humain : le partage d'un destin ${ }^{2}$}

Comment ne pas être religieux. Ce constat, à la manière de l'ouvrage récent Comment ne pas finir comme tes parents ${ }^{3}$ qui décrit une pratique, la pleine conscience, et fournit des clés pour aider l'adolescent à franchir l'étape critique du passage à l'âge adulte, annonce un manuel pour ne être pris au piège de la religion: ne pas finir religieux. Comment ne pas être religieux. Ou : comment conserver son esprit critique.

Comment ne pas être religieux ?! Phrase interrogative exclamative jusqu'à l'incitation exhortative. Comment pourrait-on ne pas être religieux?! Sous-entendu: on est nécessairement religieux, quoi qu'on fasse. Comme une injonction existentielle à laquelle l'humain ne peut que consentir.

Cette expression, comment ne pas être religieux, dit une chose et son contraire. On entend : «faisons tout pour ne pas être religieux ». Il y résonne aussi : «l'humain est nécessairement religieux. » Provocation? Ambivalence névrotique (sic Freud) de l'être religieux? Davantage: le religieux, tropisme de l'humain. Dès lors, l'humain chercherait par tous les moyens (la raison, la stratégie...) à le contrecarrer. Les Grecs,

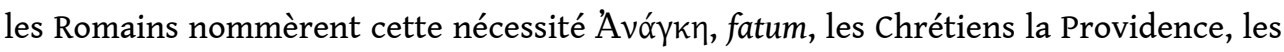
Bouddhistes notre karma. Nos contemporains athées voient dans le dieu Dollar ce tropisme transposé, sécularisé, qui trône dans la maxime américaine des billets de banque : in God we trust.

\section{Epochè des religions instituées et eidos du religieux}

Comment ne pas être religieux. Le comment dit une pratique, une manière d'être qui, en phénoménologie, dicte la description des phénomènes. Suivant cette approche, je décrirai un mode d'être, le mode d'être religieux, plutôt qu'une religion instituée. Conformément à la méthode de l'épochè, je neutraliserai les jugements pro/contra, louange ou rejet, l'injonction ou l'exclamation contenue dans la formule initiale duelle : nécessité à ne pas l'être/ nécessité à l'être. Surimposer un sens pré-donné, enjoindre à faire ou ne pas faire, être ou ne pas être, c'est ce à quoi que la phénoménologie, renouant en cela avec l'exigence socratique et sceptique, nous invite à renoncer. Je me centrerai donc sur le comment du mode d'être religieux, qui signe la singularité de la phénoménologie $e^{4}$.

Le risque a contrario de la vertu non-jugeante de la phénoménologie, c'est la neutralisation de la valeur de l'expérience. C'est alors une description trop dégagée de «l'intérêt » de l'humain, de ce qui importe, «vaut » pour lui, de ce que Husserl nomma l'attention, cet acte insigne qui donne de la valeur à mon activité perceptive ${ }^{5}$. Pour conjurer ce risque, je mène une description non formelle du religieux, mue par l'attention à la valeur du religieux pour l'humain. Mon interrogation directrice sera : l'humain se pose toujours à nouveau la question de quelque chose qui le dépasse, qui l'aimante, qui donne un sens à ce qu'il est, à ce qu'il fait, à sa vie d'humain (même, surtout s'il y résiste, y oppose de l'absurde (Camus), du cynisme (Cioran)). Le religieux et l'humain auront-ils en partage un destin?

Qu'est-ce que le religieux ? Suivant l'épochè, je fais abstraction des religions, instituées ou non. Autrement dit, je mets en suspens les contenus culturels (confessionnels, 
dogmatiques, rituels, coutumiers) ${ }^{6}$. N'étant pas niés mais neutralisés, ils nourrissent vertueusement la description non-formelle proposée. Pour ce faire, concrètement, je mène une variation eidétique de ces contenus multiples: l'eidos du mode d'être religieux qui en émane y sera gros de cette variation.

L'hypothèse que je vais explorer sur cette base s'énonce ainsi : l'humain est un être relié, un être qui, en conscience, entre en relation avec un autre que lui et s'y relie en s'y ouvrant, reconnaissant, vénérant la nature supérieure ou extérieure de cet autre.

\section{Religio : du conflit étymologique à l'unité inclusive du phénomène}

Dans De l'invention oratoire, Cicéron définit ainsi religio: «La religion est le fait de s'occuper, de se soucier d'une nature supérieure que l'on appelle divine et de lui rendre un culte (curam caerimoniamque affert) $»^{7}$.

Dans son Vocabulaire des institutions indo-européennes ${ }^{8}$, Benvéniste privilégie le sens cicéronien du terme religio. Il définit la relation entre divin et humain comme un recueil, un accueil (legere, relegere) ${ }^{9}$, et considère que le «lien " (social, de confiance, de dette) (religare, ligare) est une étymologie subséquente fantaisiste «inventé par les chrétiens $»^{10}$.

Au-delà de son autorité scientifique incontestable, le linguiste adopte une posture emblématique de l'idéologie anti-chrétienne héritée du scientisme du XIX ${ }^{e}$ siècle. C'est en effet à cette époque récente dominée par le positivisme qu'on a vainement cherché à savoir quelle était la vraie étymologie. Mais si l'on remonte à l'époque médiévale d'Augustin à Thomas, maints auteurs reprennent sans les opposer ces étymologies. Religare, ligare (relier, lier) et relegere, legere (recueillir, accueillir, recevoir) se conjuguent ensemble. Dans l'interprétation phénoménologique récente, maintes voix inviteront à neutraliser par une épochè ce débat stérile, et à penser, plutôt que la disjonction, la conjonction des racines du mot, aux fins de valoriser la complexité du phénomène religieux ${ }^{11}$. Dans ce chœur où règne un bel unisson, la voix de Derrida à la fois consonne et se distingue. Comme J. Grondin et J. Greisch il réagit contre Benvéniste en refusant d'opposer les étymologies, au motif que « rien ne se règle à la source $»^{12}:$ «Quiconque ne reconnaîtrait ni la légitimité de ce double foyer ni la prévalence chrétienne qui s'est imposée à l'intérieur même de la dite latinité devrait refuser les prémisses même d'un tel débat $»^{13}$.

Refusant le «tranchant » idéologique du linguiste, Derrida plaide selon la différance pour l'altérité irréductible des deux phénomènes. Pas d'origine une du phénomène religieux, une partition originaire qui fait sa fécondité.

Je reprends à mon compte ce geste heureux qui accorde au phénomène religieux un double foyer irréductible. Mais je l'inscris dans une dynamique plus inclusive, à l'image généreuse du vers de Gérard de Nerval dans Myrtho : «le pâle hortensia s'unit au myrte vert $\aleph^{14}$, où s'allient métaphoriquement christianisme et paganisme. Voici la formulation dynamique de cette alliance: en me recueillant, en (me) rassemblant (relegere), depuis ce mouvement prime, je me relie à l'autre (religare), entrant ainsi dans une œuvre de témoignage. Ces deux mouvements, 1. entrer-creuser en soi-même, y découvrir une ouverture interne ( $\mathrm{du}$ cœur souvent), puis 2 . se tourner vers l'autre, l'accueillir riche de ce recueil(lement), voilà une dynamique éprouvée par maints phénoménologues, qui recoupe d'ailleurs l'expérience ancienne de nombre de mystiques ${ }^{15}$. C'est cette dynamique en deux temps qui sert ici de matrice à l'exploration 
du mode d'être religieux et permet de préciser le fil directeur d'exploration du mode d'être religieux mentionné ci-dessus comme " un être relié-ouvert à un autre que soi ».

\section{Esquisse de description située du mode d'être religieux}

Cherchant l'eidos du mode d'être religieux depuis l'épochè inclusive de ses contenus institués, tablant sur l'unité du phénomène religieux par intégration de ces deux sources sémantiques, je vais à présent examiner les propriétés de la relation entre humain et divin qui y sont déposées et souvent mêlées.

Dans la définition cicéronienne de la religion, caerimonia et cura sont les mots qui caractérisent le lien entre divin (divinam) et humain. Ils désignent des qualités spirituelles-morales de vénération respectueuse liée à un culte d'une part, de soin attentif d'autre part.

Ces deux qualités forgent l'intimité d'un lien enté sur la reconnaissance d'une réalité qui me dépasse et me nourrit à la fois. Or, à côté de cette modalité relationnelle mariant soin et vénération, je voudrais mentionner une autre modalité.

Il s'agit de la croyance. Voilà une modalité relationnelle qui implique foi, confiance en cet autre que, dès lors, j'honore pour ce soutien qu'il m'offre, et qui a pour effet éventuel une demande (la dite prière de demande). C'est mutatis mutandis la modalité retenue par l'article Religion de l'Encyclopédie de Diderot-d'Alembert, qui dit : « Religion se dit plus particulièrement du système particulier de créance et de culte qui a lieu dans tel ou tel pays, dans telle ou telle secte, dans tel ou tel termes, etc. $»^{16}$.

Certes, la ligne de partage entre une relation de vénération (caerimonia) et une relation de croyance (ici créance) est ténue. Toutefois il apparaît que ces deux qualités du mode d'être religieux déterminent différemment ce dernier. Chez Cicéron, la vénération est liée à un rite, une cérémonie (un culte), et la qualité de la vénération prescrite chez la religieuse, le religieux s'inscrit dans ce cadre rituel sans engager aucune attente ni demande des personnes participant au rite. L'attitude de vénération est donnée avec et par le cadre. Par contre, la croyance signifie un engagement personnel du sujet religieux qui correspond à un état intérieur d'adhésion : cette qualité est donc moins donnée avec le rituel que vécue par le sujet.

Dans Foi et savoir, Derrida précise :

Tout acte de croyance implique un acquiescement au témoignage d'un tout autre. Ce tout autre est inaccessible, on ne peut ni le voir ni le toucher, et pourtant c'est

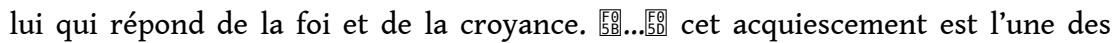

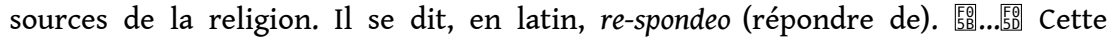
question de la réponse ou de la responsabilité 'précède' toute religion constituée. Dans toute croyan-ce, il faut d'abord se rendre à l'autre ${ }_{5 \mathrm{~B}}^{\mathrm{F} . . .500^{17}}$.

Pour Derrida, l'acte de croire est une des sources de la religion. Ma question sera pourtant : la croyance se surimpose-t-elle au mode d'être essentiellement vénérant du religieux ? La question doit être posée. La présence de la croyance ne répond-elle pas à une évolution historique du mode d'être religieux liée à un processus culturel global de subjectivation qui va de pair avec l'effacement de la puissance native du rituel liturgique ? Ou bien, dit axiologiquement : ne perd-on pas quelque chose d'essentiel au religieux quand on exhausse la croyance au détriment de la vénération ${ }^{18}$ ?

Dans le prolongement de la définition des encyclopédistes et de la construction (historique, axiologique) de la distinction entre vénération et croyance, mentionnons 
une troisième propriété du mode d'être religieux, identifiée par exemple par Durkheim dans Les formes élémentaires de la vie religieuse (1912). Le sociologue y définit la relation entre humain et divin comme une relation socio-communautaire : « Une religion est un système solidaire de croyances et de pratiques relatives à des choses sacrées 藍...㺝 croyances et pratiques qui unissent en une même communauté morale, appelée Église, tous ceux qui y adhèrent $»^{19}$.

L'auteur propose une définition du phénomène religieux en extériorité, comme pratique sociale, l'acte individuel d'adhésion croyante étant inséré dans un système communautaire nommé ici Église. Dans cette troisième propriété, on aperçoit une distinction analogue à celle (vénération/croyance) qui traverse la deuxième propriété. Selon que l'on prend communauté au sens de la charité individuelle envers le prochain ou au sens d'une cuvre commune, ensemble d'actes participant à l'unité liturgique, on insiste soit sur sa dimension interpersonnelle ou sur son être-ensemble commun ${ }^{20}$.

En réalité, les religions au sens confessionnel-théologique des trois monothéismes ou au sens anthropologique des religions dites orientales ${ }^{21}$ partagent ces trois propriétés : 1) un être relié-ouvert à autre que soi (Dieu, une réalité absolue surnaturelle) ;2) la définition de cette relation selon le mode vénération ou croyance; 3) la situation communautaire, caritative ou ecclésiale. Ces trois propriétés sont théologiquement et culturellement pertinentes. Ma question sera, à nouveau : ces deux dernières sont-elles des surimpressions attachées à l'expérience du religieux? Des propriétés accidentelles au religieux qui serait d'abord cet être relié-ouvert à autre que soi ? Ne sont-elles pas des représentations culturelles, visions du monde (Weltanschauungen) sur-imposées sur l'expérience prime du sujet? Au \$44 des Méditations cartésiennes, Husserl opère ainsi une réduction à la sphère du propre (Eigenheitssphäre) en écartant tout prédicat culturel lié à des représentations générales du sujet ${ }^{22}$. A contrario, doivent-elles être intégrées comme des variations nourrissant et incarnant l'essence du religieux ? On aura compris que mon choix sera de mettre à l'épreuve ces propriétés (vénération/croyance; ecclésialité/charité) pour évaluer si, et si oui, ce qui en elles détermine in fine le religieux.

\section{II. Éléments de phénoménologie socio-historique du religieux}

Repartons de la définition du mode d'être religieux de l'humain comme être reliéouvert à autre que lui, qui puise en cet autre la profondeur vénérante/croyante, caritative/ecclésiale de son être. Explorons depuis cette définition les critiques feuerbachienne puis marxienne de la religion.

Pourquoi faire jouer ces critiques? L'idée est de tester la pertinence de l'intégration des propriétés $n^{\circ} 2$ et 3 du religieux, vénération/croyance, ecclésialité/charité. La question étant : l'épochè intégratrice inclusive que j'ai adoptée est-elle pertinente? Si une épochè plus discriminante n'est pas retenue pour la raison qu'elle conduit à « jeter le bébé avec l'eau du bain ", parce qu'elle ne permet pas de décrire concrètement le mode d'être religieux mais le réduit à une forme sans contenu, l'épochè intégratrice n'est-elle pas à son tour insuffisamment radicale? Ne réintroduit-elle pas subrepticement des préjugés historico-culturels in-interrogés? 


\section{Lire Marx et Feuerbach à la lumière de Husserl ; lire Marx à la lumière de Feuerbach}

Marx et Feuerbach ont procédé à une critique d'une radicalité inédite de l'attitude religieuse ${ }^{23}$. Pour cette exploration redoublée, ce test du type d'épochè à appliquer pour rendre compte au plus juste du mode d'être religieux, je ferai l'hypothèse suivante: n'anticipent-ils pas, dans leur sape du religieux, l'épochè radicale des représentations culturelles? En lisant rétroactivement ces deux auteurs matérialistes au prisme phénoménologique de l'épochè, je voudrais vérifier si par leur critique même ils ne conservent pas toutefois un sens minimal du mode d'être religieux comme être reliéouvert à autre que soi. La question corrélative sera : retiennent-ils aussi quelque chose, l'un ou l'autre, des deux propriétés que j'ai retenues moi-même, vénération/croyance, ecclésialité/charité?

Mais pourquoi s'allier à Feuerbach et à Marx ? Là où les Lumières opèrent une critique culturelle qui inspire les révolutionnaires français dans leur destruction des édifices religieux, Feuerbach et Marx nous offrent une théorisation critique inédite, l'un renversant la critique initiale de l'autre. Ils emblématisent la sape la plus profonde de la base religieuse de nos sociétés occidentales et, aujourd'hui, la mondialisation aidant, de toutes les sociétés, y compris de ce qu'on appelle improprement l'Orient. C'est dans ce travail de sape que s'engouffrent à leur tour Nietzsche avec le Crépuscule des idoles $(1888)^{24}$ et Freud dans L'avenir d'une illusion (1927). ${ }^{25} \mathrm{Il}$ y a donc un enjeu déterminant à comprendre à quoi s'attaque le couple Feuerbach-Marx dans sa sape du religieux. En quoi cette sape atteint non pas l'édifice seul mais son fondement, libérant peut-être in fine un religieux sans fondement, dans son mode «non-fabriqué ».

On lit couramment le segment Feuerbach - Marx comme un emboîtement herméneutique radicalisé de la critique du religieux. Feuerbach l'initiant, Marx la poussant à bout dans son renversement ${ }^{26}$. Au motif que Feuerbach ne produirait dans sa critique de l'idéalisme théologico-religieux hégélien qu'un déplacement en faveur de la « religion » de l'humain : cette " adoration d'un dieu nouveau, le genre humain », selon la formule-choc de H. de Lubac ${ }^{27}$.

Je voudrais montrer que le diagnostic de Feuerbach est en réalité plus discernant que celui de Marx ${ }^{28}$. Pour commencer, l'assertion de F. Engels - «nous fûmes tous 痗...區 des feuerbachiens $»^{29}$ - signifie bien que la philosophie de Feuerbach, loin d'être un essai que transformera radicalement Marx, est la plate-forme matricielle depuis laquelle émerge la critique de ce dernier. Notre lecture contre-herméneutique, généalogique, remontant de Marx à Feuerbach, permettra je l'espère de cerner $a$ contrario la teneur phénoménologique radicale de leur approche du mode d'être religieux.

\section{Le mécanisme de l'Entäußerung feuerbachienne : une épochè du concept de Dieu ouvrant sur l'expérience orante du mystère}

Feuerbach est c'est bien connu l'homme d'un livre, L'essence du christianisme, qui parait en 1841, et s'annonce d'emblée comme un ouvrage fondamentalement antireligieux.

Dès 1830, il publie anonymement un premier ouvrage, Pensées sur la mort et l'immortalités ${ }^{30}$ où il critique la vision chrétienne de l'âme. Il y reprend les arguments des matérialistes, qui rejettent l'immortalité de la personne, la raison seule possédant cette dimension non-mortelle. Dans le milieu alors dominant du hégélianisme, les réactions 
ne manquent pas, qui s'effraient de l'athéisme radical de Feuerbach. Cette publication suscite une telle polémique que son auteur sera perçu par beaucoup comme un ennemi juré de la religion. Conséquence immédiate : l'ouvrage est interdit sitôt publié. En 1832, Feuerbach démissionne de sa chaire de philosophie à l'Université de Erlangen. Après plusieurs échecs, il abandonne en 1836 toute idée de carrière universitaire, et c'est dans ce contexte de solitude académique, soutenu par sa femme riche héritière, qu'il rédige son maître-ouvrage, L'essence du christianisme.

La thèse principale qui y est développée est celle-ci : l'attitude de l'humain qui croit en Dieu révèle son aliénation interne. Ainsi, les qualités humaines de liberté, conscience, imagination créatrice, y sont en réalité projetées en Dieu ${ }^{31}$. En ce sens, les déterminations divines ne sont que des déterminations humaines absolutisées:

Pour enrichir Dieu, l'homme doit s'appauvrir; pour que Dieu soit tout, l'homme doit n'être rien. 孯...區 Ce que l'homme se retire, ce dont il se prive, il n'en jouit que

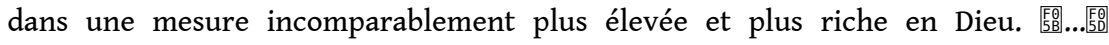
L'homme affirme en Dieu ce qu'il nie en lui-même ${ }^{32}$.

Dans les pages précédentes, l'auteur a analysé précisément comment la dite «projection» de l'homme en Dieu, en fait les représentations (Vorstellungen) idéales qu'ils s'en fait, sont pour la religieuse, le religieux, des réalités vraies. Tel est le mécanisme de la croyance: « miséricordieux pour qualifier Dieu) ne sont pas des représentations, ni des images que l'homme se fait de Dieu, différentes de ce qu'est Dieu en soi, mais des vérités, des

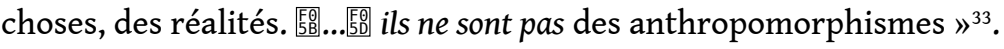

Si l'on suit le fil critique de l'analyse de Feuerbach, on dira: perdu dans ces «projections» que, s'auto-illusionnant, il prend pour la réalité vraie, l'humain se dépouille, se rend étranger à lui-même, c'est-à-dire au sens propre s'aliène (entäußert sich $)^{34}$. La tâche de la critique de la croyance en Dieu sera donc de restituer à l'homme son être perdu en Dieu.

En fait, cette analyse révèle que Feuerbach, plus que Hegel, a été le premier à user systématiquement et consciemment du concept d'aliénation dans le sens où on l'entend encore aujourd'hui, c'est-à-dire comme un concept critique. Car chez Hegel, le concept d'aliénation (Entäußerung/Entfremdung) ${ }^{35}$ est d'abord descriptif. Il répond à un moment de la dialectique : il rend compte du processus d'objectivation de l'esprit qui fait à terme émerger une altérité véritable. Bref, il est pensé comme une négativité conduite à son dépassement positif ${ }^{36}$. Quel est $a$ contrario le mécanisme original de l'aliénation chez Feuerbach?

L'auteur de L'essence du christianisme pose tout d'abord que le seul « objet » de l'humain, c'est Dieu. La conscience est la condition de possibilité à partir de quoi l'humain prend Dieu pour objet. Dieu devient alors l'« objet intérieur » de l'homme conscient: il se trouve inclus dans un être qui possède une vie subjective. En analysant la conscience, Feuerbach découvre aussi que ce qui la caractérise, son objet propre, c'est son êtresujet. Mais, loin de penser la subjectivité comme une réalité singulière vécue en première personne, il considère que l'être-sujet objet de la conscience est un être-essence, un genre. Ainsi son présupposé, encore ici marqué de hégélianisme, est qu'il ne peut y avoir d'objet de conscience que générique, universel. Ainsi l'objet de la conscience se donne sous une forme double et à deux reprises, d'une part comme Dieu, d'autre part comme être-sujet essentiel-générique. 
C'est cette réalité double de l'objet de la conscience qui constitue selon Feuerbach le cœur du mécanisme de la conscience religieuse. Car ce redoublement de l'objet de la conscience humaine, Dieu et l'être-sujet, est aussi le principe de sa confusion, ce que Feuerbach nomme son aliénation. L'humain s'illusionne en croyant vivre en Dieu ce qu'il vit en lui, dans son être-sujet. Au fond, le mécanisme de la croyance est celui d'une représentation-projection de l'homme en Dieu, qui par principe s'ignore elle-même comme telle.

Ainsi, l'homme mène une "vie double " (zweifaches Leben) qui le caractérise d'ailleurs de façon unique comme humain ${ }^{37}$. D'une part, il fait le constat de son existence finie et de la douleur que suscite cette finitude. Il est un être de chair, sensible et mortel : voilà son existence. D'autre part, il a une vie intérieure qui le fait entrer dans le règne immortel des essences. Devant la douleur de l'épreuve de la finitude, l'homme se plaît à s'échapper dans l'essence, puis à se confondre dans l'absolu que Dieu lui offre.

S'il érige pour ainsi dire ses limites en limites (Schranken) du genre, ceci repose sur

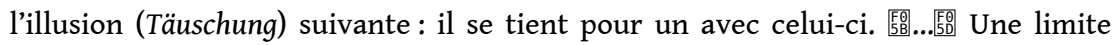

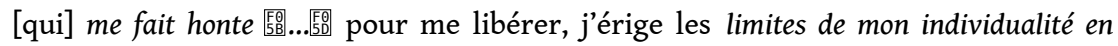
limites de l'essence humaine elle-même ${ }^{38}$.

Quand la distinction entre vie sensible individuelle et vie subjective essentielle devient poreuse jusqu'à la confusion, l'individu, envahi par la croyance qu'il est le genre, a franchi le seuil de l'illusion suprême par laquelle il va représenter son essence en Dieu, l'essence des essences. Or on l'a dit, ce «transfert» double, de l'individu au genreessence puis de l'essence humaine à l'essence divine n'est pas perçu par l'humain comme une illusion : c'est le comble de l'illusion, de s'ignorer elle-même comme telle.

$\mathrm{Au}$ fond, l'humain, par ce mécanisme par lequel il se représente en Dieu, c'est-à-dire objective sa vie hors de lui-même dans l'essence en la situant en l'essence de Dieu, espère sortir de son existence sensible limitée où il se confond encore avec l'animal. Le paradoxe est donc que, cherchant à sortir de l'indistinction animale en laquelle « la vie intérieure ne fait qu'un (eins) avec la vie extérieure $»^{39}$, l'humain retombe à son corps défendant dans une confusion à la puissance, celle où sa vie intérieure s'écrase contre la vie divine absolue.

Mais la religion offre aussi pour Feuerbach un premier essai de sortie de la confusion

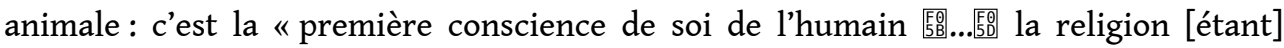
l'essence de l'enfance (kindlich) de l'humanité $\aleph^{40}$. En ce sens, il se distingue de Hegel qui fait de la religion une figure accomplie de l'absolu. Pourtant, prenant conscience de son intériorité via sa projection hors de lui en Dieu, l'humain s'objective comme genre. Il se prend pour un autre que lui et s'aliène littéralement, croyant se connaitre lui-même.

Le mécanisme de l'aliénation analysé par Feuerbach engage donc bel et bien une critique de la religion entendue comme une relation où l'humain s'absolutise illusoirement en s'identifiant à cet autre qu'est Dieu. L'humain s'objectivant lui-même en l'autre, il se perd de vue. On retrouve ici deux des trois propriétés du religieux que j'ai établies plus haut : premièrement, la relation de l'humain à autre que soi, ici à Dieu et, deuxièmement, la sous-propriété de la croyance, ici une projection-aliénation à la puissance : une perte de soi en l'autre in-sue comme perte.

Mais le point décisif est également celui-ci: on peut tout autant reprendre ce mécanisme de l'aliénation en mettant en exergue le mode de relation que l'humain entretient avec un autre qui est son altérité interne et qui le décentre de lui-même en lui offrant la dynamique première d'une "conscience de soi », celle de l'enfant par 
exemple. On obtient alors le mode d'être nodal du religieux comme être relié. Et ce mode d'être relié-ouvert correspond au religieux désencombré de sa sous-propriété de croyance-illusion.

Dans sa structure en deux parties, L'essence du christianisme fait justement ressortir ces deux plans, descriptif d'une part, critique d'autre part. La deuxième partie, intitulée "L'essence non-vraie, c'est-à-dire théologique de la religion" (das unwahre, d.i. theologische Wesen der Religion) ${ }^{41}$, répond au plan de la critique de la religion qu'on a jusqu'ici développée à travers le mécanisme de l'aliénation comme projection illusoire. Son essence non-vraie est en effet celle de son discours spéculatif sur Dieu ou "théologie». Dans une langue qui manie brillamment oxymores et antinomies, Feuerbach met la théologie en contradiction, au titre de son impossibilité à rendre raison, à expliquer l'inexplicable :

Dieu est le concept qui supplée au manque de la théorie. Il est l'explication de l'inexplicable, qui n'explique rien, puisqu'elle doit tout expliquer sans distinction il est la nuit de la théorie, qui pourtant éclaire tout pour le sentiment [Gemüt], du

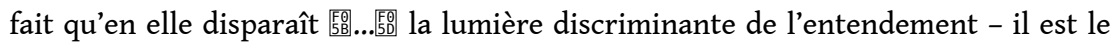
non-savoir, qui résout tout doute, puisqu'il les abat tous. Il sait tout, parce qu'il ne

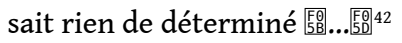

Or cette deuxième partie, qui déconstruit le rapport conceptuel fallacieux, illusoire et projectif au religieux, préparée dès l'Introduction par la description du mécanisme de l'aliénation de l'homme en Dieu qu'on a analysé, ne se comprend qu'à la lumière de la première, qui décrit justement «l'essence vraie, c'est-à-dire anthropologique de la religion" (das wahre d.i., anthropologische Wesen der Religion) ${ }^{43}$. On retient souvent de Feuerbach la seule deuxième partie critique, celle d'ailleurs sur laquelle prend appui Marx à sa suite, celle aussi qui fut seule mise au programme de l'Agrégation de philosophie en 2018. Or cette critique est incompréhensible si on la tronque de sa base descriptive. Cette dernière seule valide l'essence vraie, anthropologique, de la religion. La première partie s'avère d'ailleurs plus longue (plus de 200 pages dans le texte allemand), là où la deuxième n'en compte que 130. Comme pour nous en convaincre, après avoir attesté de l'essence anthropologique vraie de la religion, Feuerbach le redit à l'orée de la deuxième partie : "le point de vue essentiel de la religion est pratique, c'est-à-dire ici subjectif $»^{44}$.

Le fil rouge de cette partie " anthropologique » sera celui du mystère (Geheimnis) comme relation inédite de l'humain relié-ouvert en son centre à cet autre qu'est Dieu ${ }^{45}$.

En reprenant les commentaires d'Augustin, de Luther, Melanchton, Bernard, Ambroise, Clément d'Alexandrie, Origène, Tertullien, mais aussi des pensées mystiques de Boehme ou de Schelling, Feuerbach nous fait entrer dans l'essentiel du religieux. C'est l'intériorité du cœur, le vécu de la foi, les rites qui orchestrent la vie sacramentelle du mystère liturgique, et se résument dans l'acte prime de la prière : «L'acte essentiel de la religion, celui dans lequel elle confirme ce que nous désignions comme constituant son essence, est la prière ${ }^{46}$.

Ces vécus que la prière résume sont à leur tour aimantés par la puissance de l'amour, dont Feuerbach affirme, en une formule-choc: «L'amour l'emporte sur Dieu, 監...兯 il est ce à quoi Dieu a sacrifié sa majesté divine, il a déterminé Dieu à extérioriser (Entäußerung) sa divinité » ${ }^{47}$.

Notons le choix judicieux de J.-P. Osier : Entäußerung n'est plus aliénation, comme à propos du mécanisme double d'objectivation de l'humain singulier en l'essence et de son essence en Dieu, mais extériorisation. Car il y va ici de l'incarnation de Dieu sortant 
de lui-même pour entrer en l'humain, et ouvrant ainsi l'humain rassemblé à autre que soi. Dans les deux cas, il y a bien sortie de soi vers autre que soi, excentration, découverte de l'altérité à soi à même soi. Mais si l'on reprend le mécanisme d'Entäußerung en le déchargeant de sa charge critique et en lui offrant une vertu descriptive, on redécouvre la dynamique d'un soi relié à autre que soi, l'accueillant en soi (relegere) et s'ouvrant à lui (religare), selon le double mouvement du religieux que nous avons établi plus haut comme sa propriété prime.

Ainsi, Feuerbach peut dire de l'humain qu'il est religieux. Il est une conscience en soi excentrée, ouverte à l'autre en son centre, là où l'animal ne l'est pas. Au fond, la promesse des deux sens de l'expression comment ne pas être religieux, critique (aliénation théologique) et descriptif (extériorisation anthropologique), est ici tenue.

Quant aux propriétés 2 et 3 (croyance/vénération; charité/être ecclésial), la part critique du mécanisme d'Entäußerung, d'aliénation théologique, répond au processus subjectif de la croyance, représentation projective source de confusion. Quant à sa part descriptive, elle laisse indemne la dimension anthropologique d'extériorisation, qui ouvre sur l'attitude mystérique de la vénération et du rite ecclésial.

\section{L'épochè marxienne : une régression paradoxale?}

La critique marxienne de la religion comme "opium du peuple » emprunte dès lors au seul versant critique-théologique feuerbachien. Cette critique se situe au plan communautaire socio-politique, et c'est le "peuple», opprimé, qui est invité à se libérer de la catégorie dite alors « bourgeoise » de la religion : « La religion est le soupir de la créature opprimée tant que bonheur illusoire (illusorisch) du peuple est l'exigence de son bonheur réel ${ }^{48}$.

Ainsi s'exprime Marx deux ans seulement après Feuerbach. Mettant en avant le peuple, il pense d'emblée la religion au plan communautaire et politique. C'est toute la collectivité qui se trouve soumise à des conditions oppressives : la religion figure en première ligne dans sa critique de l'oppression du peuple, car elle le conditionne en l'illusionnant par un bonheur factice. C'est le sens de l'image de l'opium, cette drogue qui, endormant l'examen critique, crée l'oubli de soi.

J'ai mentionné plus haut cette troisième propriété du religieux qui correspond, à l'exemple de Durkheim, à la relation sociocommunautaire entre humain et divin. C'est depuis cette propriété de la religion que Marx mène sa critique. Le peuple y figure l'être-relié qui subit l'oppression d'un lien se faisant alors chaîne.

Aussi le sens marxien de l'aliénation, celui que subit le peuple avec une religion qui endort sa vigilance, est-il univoque. Elle le rend étranger à lui-même (entfremdet), selon le sens économique puis anthropologique de l'aliénation que déploiera Marx dans les Manuscrits de 44 puis dans Le Capital. En effet, quel que soit le plan où l'humain est aliéné, que ce plan soit psychique, social, économique, politique ou anthropologique ${ }^{49}$, le terme retenu par Marx, Entfremdung, a une charge critique univoque. Il a perdu la dimension descriptive qu'il avait chez Hegel, cette force descriptive justement aussi présente chez Feuerbach et alors liée à l'usage du terme de Entäußerung, rendu par " extériorisation ${ }^{50}$. Chez Feuerbach, on a vu que ces deux usages répondent aux deux plans, critique et descriptif de l'approche du religieux, théologique et anthropologique. Or chez Marx, si la religion est la première figure de la critique de l'aliénation de l'humain, elle est ensuite dépassée par une critique plus large de l'être séparé de soi, 
coupé de sa vie concrète. D'où une critique radicale de la conscience de soi, où l'humain s'abstrait dans un monde idéel, et une critique conjointe de l'activité du travail, où l'humain s'objective. En réalité, selon Marx, c'est l'aliénation objective (le travail) qui génère l'aliénation subjective (la conscience abstraite), non l'inverse: "Une conséquence immédiate du fait que l'homme est rendu étranger au produit de son travail, à son activité vitale, à son être générique, est celle-ci : l'homme est rendu étranger à l'homme $»^{51}$.

Notons que Marx reprend à Feuerbach nombre de formulations, parfois au mot près, en les transposant du plan religieux au plan économique. L'objet n'y est plus l'humain et Dieu, mais la marchandise et le travail : «L'ouvrier devient d'autant plus pauvre qu'il

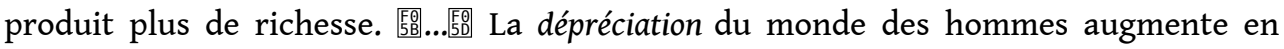
raison directe de la mise en valeur du monde des choses $»^{52}$.

Bref, alors que Marx critique le devenir étranger de l'humain à lui-même dans son activité de travailleur et dans son produit, qui l'objective et où il se perd ${ }^{53}$, Feuerbach décrit le devenir autre de l'humain à soi dans son essence et la représentation de celleci en Dieu. Alors que Marx cherche en hégélien à transformer cette aliénation pour libérer l'humain de son travail, Feuerbach voit dans la double extériorisation le processus nécessaire indépassable de la prise de conscience par l'humain de son insigne humanité.

$\mathrm{Au}$ fond, Feuerbach ferait à Marx la même critique qu'à Hegel: sur le plan anthropologique, celui de l'homme religieux, il n'y a pas de sortie de l'aliénation. Définitive, l'aliénation est la nécessité socio-historique, le destin assumé qu'humain et religion ont en partage. Dans L'essence du christianisme, seul le plan théologique dénonce l'essence inauthentique, non-vraie de la religion, qui construit sa représentation faussée, illusoire et projective ; le plan descriptif, désencombré de ces surimpressions culturelles, dévoile la quintessence religieuse dans sa visée anthropologique mystérique et rituelle, où l'humain est purement un être relié-ouvert à autre que soi.

\section{Conclusion}

A rebours de l'herméneutique qui voit en Marx la radicalisation et l'extension du mécanisme feuerbachien de l'aliénation au plan économique, j'ai montré que Feuerbach réunit par delà Marx la double exigence critique et descriptive de la phénoménologie, à savoir de l'épochè comme limitation (Einschränkung) et ouverture (Beschränkung) ${ }^{54}$. Comment ne pas être religieux. A ce constat-interrogation, Feuerbach en bon phénoménologue répond à la fois par l'affirmative et la négative. Il met ainsi au jour la nécessité du mode d'être religieux comme être relié-ouvert à autre que soi.

Quant aux propriétés deux et trois du mode d'être religieux que j'ai présentées dans leur alternative vénération/croyance et ecclésialité/charité, l'anthropologie mystérique de Feuerbach semble plus en faveur des qualités rituelles de vénération ecclésiale que des vécus intersubjectifs de croyance et de charité. Mais ce point, pour être établi, exigerait un examen plus approfondi encore de la première partie de l'Essence du christianisme... 


\section{NOTES}

1. C. Yannaras, Philosophie sans rupture, Genève, Labor et Fides, 1986, p. 225.

2. J'emprunte le mot destin à S. de Beauvoir dans Le deuxième sexe (1949), Paris, Gallimard, 2018, qui refuse l'idée de destin préétabli lié à une nature, une essence, et le repense comme une nécessité socio-historique vécue à laquelle on consent. Je pense le rapport de l'humain à la religion comme un tel destin.

3. S. Michelot, A. Assier, Comment ne pas finir comme tes parents : la méditation pour les 15-25 ans, Paris, Arènes, 2016. Cf. B. Jakubowicz, Neuropsychiatrie de l'enfance et de l'adolescence, vol. 66, $\mathrm{n}^{\circ}$ 7-8, p. 476.

4. Cf. Sextus Empiricus, Esquisses pyrrhoniennes, Paris, Seuil, 1997; P. Couissin, "L'origine et l'évolution de l'épochè », Revue des études grecques, 42, 1929, p. 373-97 ; N. Depraz, Epokhè, B. Cassin éd., Vocabulaire européen des philosophies, Paris, Seuil, 2004, p. 366 sq.

5. E. Husserl, Phénoménologie de l'attention (Hua XXXVIII, 2004), Paris, Vrin, 2009, trad. par N. Depraz ; N. Depraz, Attention et vigilance. A la croisée de la phénoménologie et des sciences cognitives, Paris, P.U.F., 2014, Partie II.

6. Quelques exemples indicatifs : dogme de la Trinité, pratique de la prière, culte voué à Marie

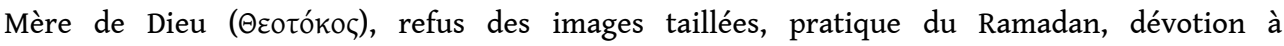
Avakokiteshvara le bodhisattva de la compassion dans le Bouddhisme ou bien à Mahakala, déesse à six bras dans le Bouddhisme vajrayana, équivalente à Shiva dans l'Hindouisme.

7. Cicéron, De l'invention (84-83), Paris, Les Belles Lettres, 1994, LII De l'orateur, 53, p. 161.

8. E. Benvéniste, Le vocabulaire des institutions indo-européennes, II, Paris, Minuit, 1969, L3 « La religion ", p. 265 sq.

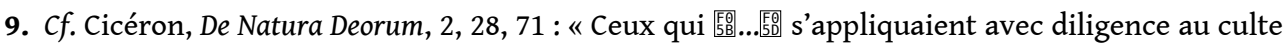
des dieux, en le reprenant et en le relisant, méritaient le qualificatif de religieux qui vient de relire (religiosi ex relegendo) ${ }_{56}$....5. religieux ».

10. E. Benvéniste, op. cit., p. 265. Pour J. Grondin, Benvéniste privilégie l'étymologie romaine pour critiquer la vision chrétienne (La philosophie de la religion, Paris, PUF, 2009, p. 70-71).

11. J. Greisch, Le buisson ardent et les lumières de la raison I, Paris, Cerf, 2002, p. 15 sq. Pour lui, la double étymologie livre la matrice de toute religion. Aussi J. Grondin, op. cit., p. 74.

12. J. Derrida, Foi et savoir (1996), Paris, Seuil, 2001, p. 49-59. Pour la citation, cf. p. 58. Aussi p. 47-48.

13. Op. cit., p. 59 .

14. G. de Nerval, "Les Chimères » (1854), in Les Filles du feu - Les Chimères, Paris, GarnierFlammarion, 2011.

15. N. Depraz suit ce fil dans Transcendance et incarnation. L'intersubjectivité comme altérité à soi chez Edmund Husserl, Paris, Vrin, 1995 : l'altérité à soi y est l'expérience prime d'ouverture de l'ego gage d'une relation réceptive à l'autre (au sens husserlien d'une passivité active). L'interprétation s'appuie sur les manuscrits de Husserl sur l'intersubjectivité et trouve un écho dans le concept ricœurien d'altérité interne thématisé dans Soi-même comme un autre (Paris, Seuil, 1990). Cette expérience de dissolution d'un soi-substance, prémisse de l'ouverture à l'autre est au cœur de maintes épreuves spirituelles en Orient, en Occident. Cf. par exemple J.-M. Guyon, Torrents (1863), Grenoble, Millon, 1992 ; Shântideva. Bodhicaryâvatâra. La marche vers l'éveil (VIII $\left.{ }^{\mathrm{e}}-\mathrm{IX}{ }^{\mathrm{e}} \mathrm{s}\right)$, Plazac, Ed. Padmakara, 2006-2007.

16. Je souligne. D. Diderot, J. d'Alembert, Encyclopédie ou Dictionnaire des Arts, des Sciences et des Métiers (1751-1772), Ed. numérique Coopération CNRS/Université de Chicago-ARTFL: http:// encyclopedie.uchicago.edu, vol. XIV, p. 74. 
17. J. Derrida, Foi et savoir, op. cit., p. 49-51.

18. Ces deux modalités du religieux sont de facto apparus à des moments différents : l'un définit le religieux comme une attitude de vénération donnée dans et par le rite. Il caractérise par exemple le mode d'être chrétien orthodoxe. Cf. C. Andronikof, Le sens de la liturgie, Paris, Cerf, 1988, A. Scrima, «Essai sur la spiritualité liturgique de l'Eglise d'Orient », Contacts. Revue française de l'Orthodoxie, 2018, L. Ouspensky, La théologie de l'icône dans l'Église orthodoxe, Paris, Cerf, 1980/2003. L'autre se définit par l'acte de croire qui est adhésion personnelle du sujet autonome. Initié au $\mathrm{XVI}^{\mathrm{e}}$ siècle avec la Réforme, il conduit à un processus d'individualisation par la foicroyance (Glauben). Il se construit autour du principe de la sola fide (seule la foi), doctrine du salut par le croyant. Cf. M. Weber, L'Éthique protestante et l'esprit du capitalisme, Paris, Plon, 1964/2010.

19. E. Durkheim, Les formes élémentaires de la vie religieuse (1912), Paris, Livre de Poche, 1991, p. 104.

20. Illustrons cette distinction par l'attitude caritative catholique d'une part, par l'être ecclésial orthodoxe d'autre part, où la liturgie aimante les participants. Cf. J.-M. Marion, Prolégomènes à la charité (1986), Paris, La différence, 2007 ; K. Gamber, La Liturgie du rite des Gaules Icône de la Liturgie céleste, Paris, Cerf, 2019, trad., introd., comm, par N. Depraz.

21. Si le terme de théologie ne s'applique pas en Orient pour caractériser la relation de l'humain à un principe transcendant/absolu (qu'il s'agisse de l'hindouisme, du bouddhisme ou du taoïsme), celui de religion en revanche répond parfaitement. Au-delà de l'orientalisme du XIX ${ }^{\mathrm{e}}$ siècle qui imposa l'expression de "religion orientale » ou de son application aux cultes païens grécoromains au début du $\mathrm{XX}^{\mathrm{e}}$ siècle (F. Cumont, Les religions orientales dans le paganisme romain (1906), Toulouse, C. Bonnet, 1929), on reconnaît aujourd'hui que l'expression englobe les cultes d'Asie, notamment l'Inde et la Chine (cf. "Les religions orientales » in : La religion. Un enjeu pour les sociétés, Sciences humaines, été 2003).

22. E. Husserl, Cartesianische Meditationen (1950), Hua I, trad. par M. De Launay, Méditations cartésiennes, Paris, PUF, 2001.

23. L. Feuerbach, Das Wesen des Christentums, Stuttgart, Reclam, 2011, trad. par J.-P. Osier ; L'esprit du christianisme (1841), Paris, Gallimard, 2015 [abrégé WC et EC]; K. Marx-F. Engels, Kritik der Hegelschen Staatsphilosophie in Werke, Berlin, K. Dietz Verlag, 1976 [http://www.mlwerke.de/me/ me01/me01_378.htm], trad. par V. Béguin, A. Bouggard, P. Guerpillon et F. Nicodème, avec une postface de J.-F. Kervégan, Contribution à la Critique de la philosophie du droit de Hegel (1843), Paris, Les éditions sociales, 2018.

24. F. Nietzsche, Crépuscule des idoles, Paris, Folio, 1988.

25. S. Freud, L'avenir d'une illusion, Paris, Cerf, 2012.

26. P. GUILLOT, «La critique de la religion chez Feuerbach», Droits, vol. 65, no. 1, 2017, p. 147-161; L. Althusser, "Sur Feuerbach», cours sur L'Idéologie allemande (1967) in Écrits philosophiques et politiques, Paris, Stock, 1995-1997, t. II ; Pour Marx, Paris, Maspero, 1968 ; J.-P. Osier, Présentation de sa traduction française de L. Feuerbach, L'essence du Christianisme, op. cit., p. 70-71.

27. H. de Lubac, Le drame de l'humanisme athée (1944), UGE, 10/18, 1963, p. 111.

28. Dans son article «L'anthropologie comme philosophie », Methodos, 5 |2005, https://doi.org/ $10.4000 /$ methodos.320, P. Sabot ne va pas jusque-là, mais il questionne la position d'intermédiaire de Feuerbach 'entre' Hegel son critique, et Marx son précurseur.

29. F. Engels, L. Feuerbach et la fin de la philosophie classique allemande (1886), Paris, Editions sociales, 1979, p. 27.

30. L. Feuerbach, Pensées sur la mort et l'immortalité, Paris, Cerf, 1991.

31. Notons que, à ma connaissance, le concept de projection n'est pas feuerbachien, bien qu'il soit couramment utilisé dans la littérature. Par exemple, J.-C. Monod, "Infinité, immortalité, sécularisation : constitution et retraduction de contenu de la religion chrétienne chez Feuerbach ", in P. Sabot (éd.), Héritages de Feuerbach, Lille, Septentrion, 2008, p.145-160; M. 
Gauchet, Le désenchantement du monde. Une histoire politique de la religion, Paris, Gallimard, 1985. Feuerbach quant à lui parle d'objectivation, de représentation, de présentification et d'extériorisation. Projection résulte sans doute d'une lecture rétroactive, politique et/ou freudienne (cf. S. Freud, « Nouvelles remarques sur les psychonévroses de défense » (1896) in Névrose, psychose et perversion, Paris, PUF, 1985). En fait, le mécanisme de l'extériorisationaliénation de l'homme en Dieu est sans doute autant descriptif que critique.

32. EC, p. 143-144; WC, S. 71-72.

33. EC, p. 143 ; WC, p. 70. Cf. I. Garo, «La représentation chez Feuerbach. Sensation, religion et philosophie ", Archives de philosophie, 2001/4, t. 64, p. 669-693.

34. EC, p. 149 ; WC, p. 78.

35. G. W. F. Hegel, La phénoménologie de l'esprit (1807), Paris, Aubier, 1941, t.2, p. 49, n.85.

36. Cf. F. Fischbach, "Transformations du concept d'aliénation. Hegel, Feuerbach, Marx », Revue germanique internationale, 8, 2008, p. 93-112, ici p. 98-99, qui adopte globalement la lecture althusserienne d'un Feuerbach régressif par rapport à Hegel car abandonnant de ce dernier la théorie de la négativité (l'histoire, la culture, le travail), et pensant l'aliénation comme une méconnaissance illusoire de soi fondée sur la confusion entre soi et l'autre (humain et divin), prémisse de la conception marxienne de l'aliénation réelle.

37. EC, p. 117 ; WC, S. 37-38: «La religion suppose la différence essentielle de l'homme et de

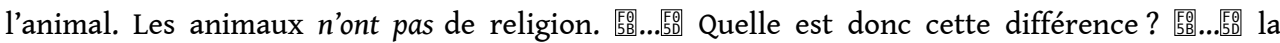
conscience, mais la conscience au sens strict [différente du 'sentiment de soi' que possède l'animal], là où un être a pour objet son genre. »

38. EC, p. 123-124; WC, S. 45.

39. EC, p. 117-118; WC, S. 38.

40. EC, p. 130 (trad. modifiée); WC, S. 53 : kindlich est traduit par Osier par infantile, ce qui lui donne un sens péjoratif que l'allemand ne contient pas, qui répond davantage à kindisch.

41. EC, p. 329-435, p. 329 ; WC, S. 284-413, S. 284.

42. EC, p. 339-340 ; WC, S. 295. Ici s'annonce précocement un geste double qui sera celui-là même de $\mathrm{M}$. Heidegger dans la réduction onto-théologique de Dieu à un concept et dans sa libération d'une théologie de la foi irréductible à tout fondement en raison. Feuerbach va d'une certaine manière, par avance, plus loin. Il identifie plus explicitement que Heidegger la vertu de la seule théologie qui vaille, celle du «non-savoir »: la théologie apophatique d'un Grégoire de Nysse, d'un Denys l'Aréopagite. (A propos de l'explicitation de ce lien implicite chez Heidegger avec la théologie aphophatique, $c f$. C. Yannaras, De l'absence et de l'inconnaissance de Dieu. D'après les écrits aréopagitiques et Martin Heidegger (Athènes, 1965), Paris, Cerf, 1971.) Feuerbach propose on va le voir une description de la pratique concrète de l'expérience religieuse, là où Heidegger, hormis dans sa précoce et luthérienne Phénoménologie de la vie religieuse (1918-1921, GA 60, Paris, Gallimard, 2011), ne s'aventura guère plus tard. $C f$. S. J. Arrien, «Foi et indication formelle, Heidegger, lecteur de saint Paul (1920-1921)», in S. J. Arrien et S. Camilleri (dir.), Le jeune Heidegger (1909-1926). Herméneutique, phénoménologie, théologie, Paris, Vrin, 2011, p. 155-172.

43. EC, p. 151-329; WC, S. 80-284.

44. EC, p. 331 ; WC, S. 284.

45. La description anthropologique du mode d'être religieux y est polarisée par la relation mystérique à Dieu dans ses différentes figures. Il n'est que de parcourir la table des matières pour s'en convaincre, qui ouvre par le mystère 10 chapitres sur 20 : mystère de l'Incarnation, du Dieu souffrant, de la Trinité, de la Mère de Dieu, du logos, de l'image divine, du principe créateur, de la nature en Dieu, de la Providence, de la Création ex nihilo, de la foi, du miracle, de la Résurrection, de la naissance surnaturelle, du Christ.

46. EC, p. 340 ; WC, S. 295. Cf. A. Steinbock, Phenomenology and Mysticism. The Verticality of Religious Experience, Indiana Press, 2007, N. Depraz, Le corps glorieux. Phénoménologie pratique de la Philocalie des Pères du désert et des pères de l'Eglise, Bruxelles, Peteers, 2008, S. Camileri, « Une nouvelle ère de 
la phénoménologie de la religion? Sur les récents travaux de Natalie Depraz et de Anthony J. Steinbock ", Meta. Research in Hermeneutics, Phenomenology and Practical philosophy, vol. IV, $\mathrm{N}^{\circ} 1$, 2012, p. 166-212.

47. EC, p. 176 ; WC, S. 106. Cette conception de l'amour comme épochè de Dieu dissout le caractère convenu de l'affirmation chrétienne «Dieu est amour » et annonce les diagnostics radicaux de Nietzsche et de J.-L. Marion dans Prolégomènes à la charité et Dieu sans l'être.

48. K. Marx, Kritik der Hegelschen Staatsphilosophie, op. cit., p. 378-379, trad. par V. Béguin, A. Bouggard, P. Guerpillon et F. Nicodème, avec une postface de J.-F. Kervégan, Contribution à la Critique de la philosophie du droit de Hegel (1843), Paris, Editions sociales, 2018.

49. Cf. S. Haber, L'aliénation: Vie sociale et expérience de la dépossession, Paris, PUF, 2007.

50. Hegel et Marx usent en allemand des deux termes sans distinction de sens. La différence passe entre dans le sens, descriptif pour l'un, critique pour l'autre, et dont Feuerbach reprend la double postulation.

51. K. Marx, Manuscrits de 1844. Économie politique \& philosophie, Paris, Editions sociales, 1972. Lien : http://classiques.uqac.ca/classiques/Marx_karl/manuscrits_1844/Manuscrits_1844.pdf, p. 62, abrégé M44.

52. M44, p. 56.

53. M44, p. 59.

54. E. Husserl, L'idée de la phénoménologie (1907) Hua II ; Paris, P.U.F., 1997, leçon 2. 